\title{
Changes in Vertical Dimension of Complete Dentures due to Rebasing with Different Techniques
}

\author{
Shammas Mohammed, Rama Krishna Alla, Achut Devarhubli, SK Shakeel
}

\begin{abstract}
Dental literature is devoid of information in respect of altered incisal pin relation due to compression molding in a single denture. It is to be reckoned that in rebasing technique, the entire segment of teeth remain fixed through a matrix of set polymethylmethacrylate that binds all the teeth. Thus, the artificial teeth do not remain as individual but act as a single unified unit of all the teeth. In view of lack of information in change in vertical dimension due to rebasing procedures and with the aim of determining the method of rebasing that result in minimal changes in occlusal discrepancy, the study was undertaken to evaluate the changes in vertical dimension of occlusion after rebasing using two different methods.
\end{abstract}

Keywords: Rebasing, Polymethylmethacrylate, Incisal pin, Articulator.

How to cite this article: Mohammed S, Alla RK, Devarhubli A, Shakeel SK. Changes in Vertical Dimension of Complete Dentures due to Rebasing with Different Techniques. World J Dent 2013;4(2):96-99.

Source of support: Nil

Conflict of interest: None declared

\section{INTRODUCTION}

It has been observed that the commonly followed compression molding procedure causes migration ${ }^{1-5}$ of artificial teeth in the mold. The resultant shift adversely affects the planned occlusion which is manifested by the loss of incisal pin contact with the incisal table of the articulator. In this respect dental literature gives ample information on the variables associated with such migration of teeth thereby stressing the importance of control $^{6-11}$ of these variables.

One such area of prosthodontic treatment and interest is rebasing for complete dentures. Rebasing is a process of refitting a denture by replacing the denture base material without changing the occlusal relations of the teeth. Rebasing may be necessary when the existing denture base resin is too light or dark in color for the patient. It may also be required if a newly processed denture exhibits porosity.

\section{MATERIALS AND METHODS}

For the purpose of convenience the methods adopted in this project have been described under the following captions.

a. Fabrication of dentures

b. Rebasing techniques

\section{Fabrication of Dentures}

Sixty test dentures were fabricated which were identical in all respects.

\section{Rebasing Techniques}

The models with overlying dentures were indexed for the purpose of reorienting subsequent to rebasing procedure. For this purpose mean value articulator with an anterior stop on the flat incisal table was selected and maxillary denture was secured to the upper member of the articulator in a standard manner. The dental stone index of occlusal surface and incisal edges were prepared. Care was taken to record only the occlusal surface and incisal edges of teeth. Sixty such dentures were indexed in a similar way.

A set of 30 dentures grouped as A1 to A30 were subjected to elimination of denture base resin by using fine fissure bur and separating disks. The procedure was carried out to leave a string of artificial teeth joined together by minimal amount of denture base matrix. The space so left between the master model and teeth was treated with modeling wax to complete the waxed form. This was finished and polished before preparation of the mold for rebasing technique. Flasking and wax elimination was done identically and the empty molds were packed using fresh polymethylmethacrylate resin mix and these were subjected to curing cycles in an acrylizer. The dentures were recovered after bench cooling overnight. The dentures along with the master model were reoriented to the articulator with the help of the index. Observations on the rise of incisal pin from the incisal table were made using a traveling microscope.

In the subsequent rebasing technique for 30 dentures grouped as B1 to B30 the models were separated from the articulator and the models were invested in the lower section of the flask. Addition silicone in the form of putty was mixed and made to cover facial and palatal surfaces of artificial teeth leaving the occlusal surfaces and incisal edges of these teeth uncovered. A dental stone counter pour was done. The two sections were separated after the dental stone was set.

Dentures were separated from the model and segment of teeth were also separated from the model and base as said earlier. The strings of teeth were relocated into its corresponding index and packing of this mold and polymerization was carried out in a standard manner. The recovered dentures along with the models after bench cooling were reoriented back on the articulator and the incisal pin opening from the incisal table (Fig. 1) in each test specimen were noted as described earlier under the 


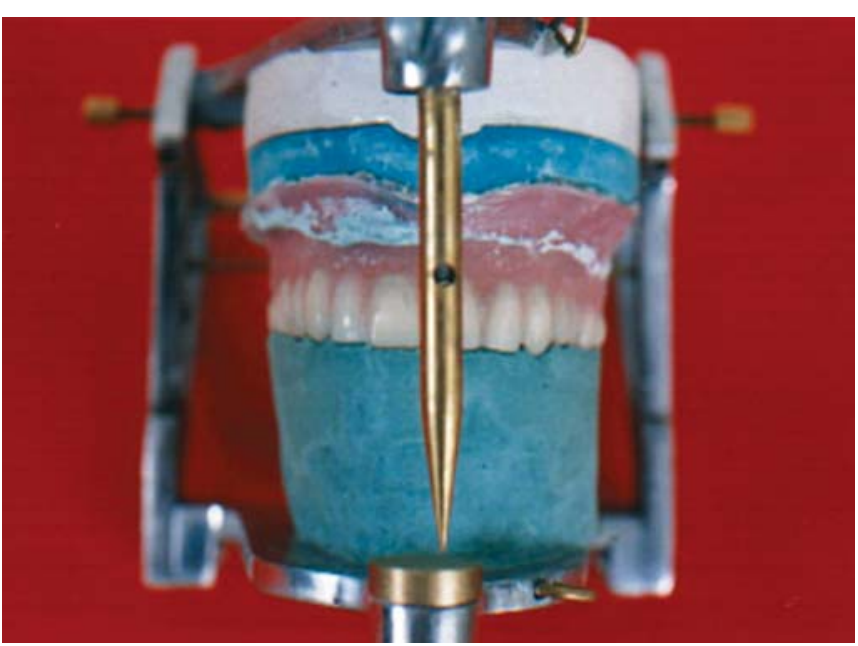

Fig. 1: Rise of incisal pin on the articulator

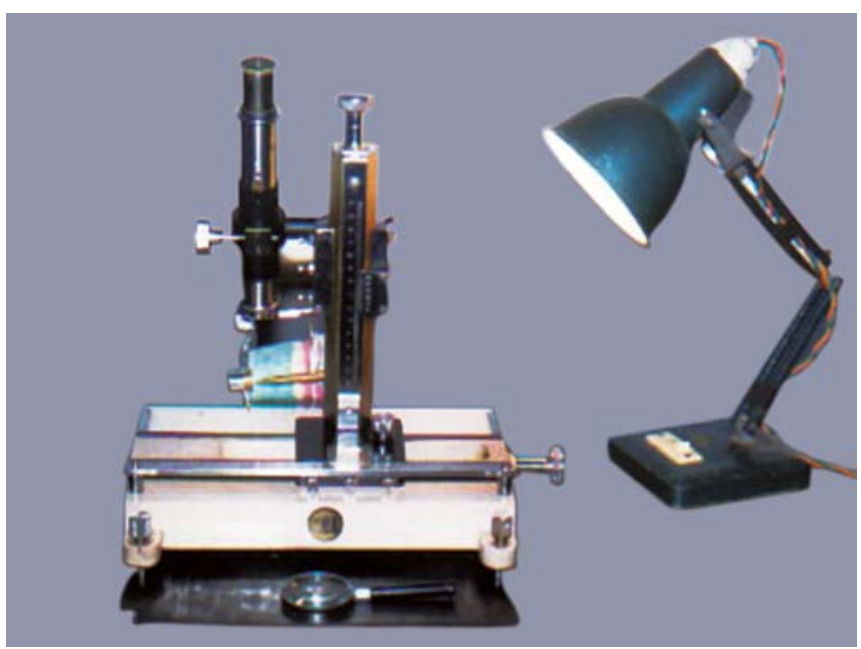

Fig. 2: Checking the amount of incisal pin rise with the help of a traveling microscope

traveling microscope (Fig. 2). Mean and standard deviation were calculated and were subjected to statistical analysis (Students t-test, unpaired and one-way ANOVA test). The findings of the study as well as statistical analysis have been presented in tabulated form. All the measurements were calculated using a traveling microscope having a least count of $0.001 \mathrm{~cm}$.

This table shows statistical comparison (Student's t-test unpaired) of the amount of rise of incisal pin from the incisal table with both the methods.

Statistical comparison (Student's t-test, unpaired) of amount of rise of incisal pin from the incisal table with both the methods (Graph 1).

\begin{tabular}{llllll}
\hline Region & $\begin{array}{c}\text { Mean } \\
(\mathrm{mm})\end{array}$ & $\begin{array}{l}\text { Standard } \\
\text { deviation }\end{array}$ & t-value & $p$-value & Significance \\
\hline $\mathrm{A}$ & 1.530 & 0.06510 & 12.87 & 0.0000 & $\mathrm{HS}$ \\
$\mathrm{B}$ & 1.296 & 0.07514 & & & \\
\hline
\end{tabular}

HS: Highly significant

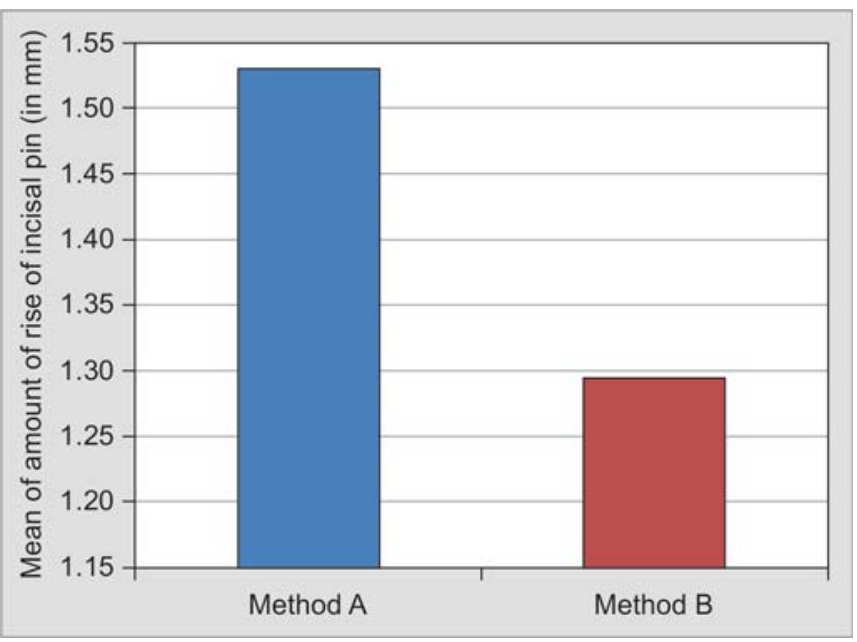

Graph 1: Amount of rise of incisal pin from the incisal table with both the methods

\section{DISCUSSION}

Rebasing involves refitting a denture by replacing the denture base material without changing the occlusal relations of the teeth. Rebasing may be needed if the patient is not happy with the color of the existing denture base or if the processed denture exhibits porosity. Since a rebased denture is of polymethylmethacrylate also, it undergoes the same processing errors due to polymerization shrinkage as it is in routine complete denture processing thereby affecting the changes in occlusion.

Hooper, ${ }^{12}$ used a Hooper duplicator to rebase the dentures, Morrow, Rudd and Rhoads ${ }^{13}$ used a reline jig, an articulator and a denture flask to rebase the dentures. Various other authors ${ }^{14-16}$ have either used an articulator or a denture flask to rebase a denture.

If at all there is plethora of doubt in mind with regards to the shift of teeth in processed dentures, it has been addressed in this project work. A number of variables in respect of changes in occlusion ${ }^{1,3-5,17-22}$ have been widely debated.

According to the studies conducted by Nelson, Kotwal and Sevedge ${ }^{4}$ and Strohaver ${ }^{5}$ the rise of incisal pin produced by compression molding procedures were under $1 \mathrm{~mm}$. But the present study which was conducted showed that the incisal pin opening was found to be between 1.25 and $1.55 \mathrm{~mm}$. Though the magnitude of change in incisal pin opening seems to be higher it may be attributed to occlusal changes due to elimination of existing denture base, changes in index either on the articulator or in the dental flask or dimensional changes in silicone putty. This is in addition to the occlusal changes induced by polymerization shrinkage by polymethylmethacrylate.

However, when the two methods of rebasing were compared for the amount of incisal pin rise from the incisal table it was found to be statistically highly significant 
(t-value: 12.87, p-value: 0.0000, at 1\% level of significance) as shown in the table. Method B (putty occlusal index) which had less amount of incisal pin rise from the incisal table was better but only marginally than method A when the mean values were compared. Method B produced less change in occlusion (reflected by incisal pin rise from the incisal table).

Method B (putty occlusal index) was easy and a convenient method and produced less amount of incisal pin rise from the incisal table though the cost factor of using putty impression material may be a disadvantage. It is recommended for the clinicians that this method may be followed routinely. The magnitudes of changes in occlusion represented by rise of vertical incisal pin are according to the materials and method used in the study and may vary if any of the parameters or materials and methods are altered.

\section{SUMMARY AND CONCLUSION}

Rebasing requires complete elimination of existing denture base leaving only a string of teeth. The procedure of rebasing could be accomplished by indexing either using an articulator or a dental flask, followed by flasking in a conventional manner. These methods employed during rebasing may result in changes in vertical dimension to the foundation. The evaluation of the two methods (method A and method B) in respect of these parameters was studied by rebasing 30 dentures employing each technique.

As all the 60 dentures were indexed using dental stone on an articulator, after rebasing, they were put back on the articulator to check for the amount of incisal pin rise from the incisal table.

When the two methods were compared for the amount of incisal pin rise from the incisal table method B showed less amount of incisal pin rise than method $\mathrm{A}$.

Based on the observations, as well as results of this study the following conclusions were drawn:

1. Both the methods resulted in rise of incisal pin from the incisal table after evaluation of rebased dentures on the articulator.

2. Method $B$ produced less rise in incisal pin opening (mean value $=1.296 \mathrm{~mm}$ ) as compared to method $\mathrm{A}$ (mean value $=1.530 \mathrm{~mm}$ ). The variation between rise in incisal pin resulting due to methods A and B for rebasing was statistically highly significant.

3. Method B, i.e. use of silicone putty index directly during flasking, was found to be convenient, less time consuming method and also resulted in less rise of incisal pin, indicating less changes in occlusal relationship to the foundation.

4. It is recommended that method B (putty occlusal index) method may be preferred over method A (dental stone occlusal index) of rebasing.

\section{REFERENCES}

1. Dukes BS, Fields H Jr, Olson JW, Scheetz JP. A laboratory study of the changes in vertical dimension using a compression molding and pour resin technique. J Prosthet Dent 1985;53: 667-69.

2. Garfunkel E. Evaluation of dimensional changes in complete dentures processed by injection-pressing and the pack-and-press technique. J Prosthet Dent 1983;50(6):757-61.

3. Mahler DB. Inarticulation of complete denture processed by the compression molding technique. J Prosthet Dent 1951;1: 557-59.

4. Nelson MW, Kotwal KR, Sevedge SR. Changes in vertical dimension of occlusion in conventional and microwave processing of complete dentures. J Prosthet Dent 1991;65: 306-08.

5. Strohaver RA. Comparison of changes in vertical dimension between compression and injection molded complete dentures. J Prosthet Dent 1989;62:716-18.

6. Kotwal KR, Monotoya ML, Charles C. Minimizing incisal pin opening during complete denture processing. J Prosthet Dent 1998;60:642-43.

7. Lerner H, Pfeiffer KR. Minimum vertical occlusal changes in cured acrylic resin dentures. J Prosthet Dent 1964;14:294-97.

8. Pera FL. Avoiding the increase of the vertical dimension of dentures in processing. J Prosthet Dent 1968;19:364-69.

9. Rudd KD. Processing complete dentures without tooth movement. Dent Clin North Am 1964;21:675-91.

10. Shippee RW. Control of increased vertical dimension of compression-molded dentures. J Prosthet Dent 1961;11: 1080-85.

11. Vig RG. Method of reducing the shifting of teeth in denture processing. J Prosthet Dent 1975;33:80-84.

12. Hooper BL. Rebasing or duplicating dentures: A method of restoring facial contour and correcting faulty retention. Dent Digest 1932;38:206-13.

13. Morrow RM, Rudd KD, Rhoads JE. Dental laboratory procedures: Complete dentures (2nd ed). St. Louis: The CV Mosby Company 1986;1:377-82.

14. Hardy IR. Rebasing the maxillary denture. Dent Digest 1949;55:23-27.

15. Kotwal KR, Houstan JC. An alternate simplified technique for rebasing complete dentures. J Prosthet Dent 1985;54:598-99.

16. Skinner EW. Acrylic denture base materials: Their physical properties and manipulation. J Prosthet Dent 1951;1:161-67.

17. Antonopoulos AN. Dimensional and occlusal changes in fluid resin dentures. J Prosthet Dent 1978;39(6):605-15.

18. Grant AA. Effect of the investment procedure on tooth movement. J Prosthet Dent 1962;12:1053-58.

19. Mainieri ET, Boone ME, Potter RH. Tooth movement and dimensional change of denture base materials using two investment methods. J Prosthet Dent 1980;44(4):368-73.

20. Perlowski SA. Investment changes during flasking as a factor of complete denture malocclusion. J Prosthet Dent 1953;3: 497-99. 
21. Wesley RC, Henderson D, Frazier QZ, Rayson JH, Ellinger CW, Lutes MR, et al. Processing changes in complete dentures: Posterior tooth contacts and pin opening. J Prosthet Dent 1973;29:46-53.

22. Zakhari KN. Relationship of investing medium to occlusal changes and vertical opening during denture construction. J Prosthet Dent 1976;36: 501-09.

\section{ABOUT THE AUTHORS}

\section{Shammas Mohammed (Corresponding Author)}

Professor, Department of Prosthodontics, Sri Rajiv Gandhi College of Dental Sciences, Bengaluru, Karnataka, India, e-mail: shammasm@rediffmail.com

\section{Rama Krishna Alla}

Assistant Professor, Department of Dental Materials, Vishnu Dental College, Bhimavaram, Andhra Pradesh, India

\section{Achut Devarhubli}

Professor, Department of Prosthodontics, Sri Rajiv Gandhi College of Dental Sciences, Bengaluru, Karnataka, India

\section{SK Shakeel}

Professor, Department of Prosthodontics, Best Dental Science College Madurai, Tamil Nadu, India 